\title{
Editorial
}

Monatsschr Kinderheilkd 2020 $168: 573$ https://doi.org/10.1007/s00112-020-00943-7 Online publiziert: 29. Mai 2020

(c) Springer Medizin Verlag GmbH, ein Teil von Springer Nature 2020

Liebe Kolleginnen und Kollegen,

ein zentrales Anliegen unserer Fachgesellschaft Deutsche Gesellschaft für Kinder- und Jugendmedizin (DGKJ) ist es, die wissenschaftliche Pädiatrie im internationalen Kontext zu stärken und den wissenschaftlichen Austausch auf hohem Niveau voranzubringen. Das war ein wesentlicher Grund, 2013 gemeinsam mit dem Springer-Verlag das internationale Open Access Journal Molecular and Cellular Pediatrics (MolCellPed) zu gründen, und ist gerade in der gegenwärtigen Zeit sehr aktuell.

Molecular and Cellular Pediatrics repräsentiert die Pädiatrie als Einheit, mit Anerkennung der hohen Interdisziplinarität der pädiatrischen Subdisziplinen. Schwerpunkt sind Grundlagenforschungs- und Originalarbeiten aus der Pädiatrie und aus allen relevanten Subdisziplinen. Ebenso werden aussagekräftige exzellente Kasuistiken, Übersichtsarbeiten und Reviews publiziert.

Die Wahrnehmung unserer Zeitschrift international ist sehr gut und bestärkt uns darin, den eingeschlagenen Weg mit Ihnen gemeinsam weiterzugehen.

So kamen 2019 beispielsweise 31\% der Artikel-Downloads aus Nordamerika und $29 \%$ aus Asien und dem Pazifikraum. Diese internationale Wahrnehmung von MolCellPed ist sehr vielversprechend. Nun sind wir alle gefordert daran mitzuwirken, dass sich dieser Impact auch in internationalen Datenban-

Ingeborg Krägeloh-Mann' für die Deutsche Gesellschaft für Kinder- und Jugendmedizin, Berlin

'Deutsche Gesellschaft für Kinder- und Jugendmedizin e. V. (DGKJ), Berlin, Deutschland

\section{Molecular and Cellular Pediatrics: ein deutsches Forum für international sichtbare pädiatrische Forschung}

ken niederschlägt. Erfolgreich sein können wir nur gemeinsam!

Bitte unterstützen Sie uns und publizieren Sie zu allen Themen der Kinderund Jugendmedizin: Reichen Sie Originalarbeiten, Minireviews zu einer innovativen Hypothese, einem aktuell diskutierten Thema oder einer interessanten Idee ein. Auch Fallberichte interessieren, wenn sie z. B. seltene Erkrankungen betreffen oder neue Aspekte zur molekularen Grundlage dargestellt werden. Ebenso freuen wir uns aufbiomarkerorientierte klinische Studien, ggf. deren Pilotdaten.

Ich möchte auch noch einmal alle Habilitanden einladen, den Inhalt ihrer Habilitationsschrift in Form eines Minireviews in MolCellPed zu publizieren. Wenn uns dies in der Mehrheit der Fälle gelingen wird, dann wäre dies eine deutliche Markierung der Forschung an deutschen Kinderkliniken in der internationalen Literatur.

Detaillierte Informationen zu Manuskripterstellung und -einreichung finden Sie unter: www.molcellped. springeropen.com. Die DGKJ übernimmt die Publikationsgebühren, sodass für die Autoren keine Kosten anfallen.

Ich danke Ihnen für Ihre Unterstützung und freue mich auf Ihre Einreichungen.

Mit herzlichen Grüßen

Ihre

Ingeborg Krägeloh-Mann

Präsidentin der DGKJ

\section{Korrespondenzadresse}

Prof. Dr. med. Ingeborg Krägeloh-Mann

Deutsche Gesellschaft für Kinder- und

Jugendmedizin e.V. (DGKJ)

Chausseestr. 128/129, 10115 Berlin,

Deutschland

info@dgkj.de

Interessenkonflikt. I. Krägeloh-Mann gibt an, dass kein Interessenkonflikt besteht. 